\title{
Neutron scattering investigation of high-frequency dynamics in glassy glucose
}

\author{
N. Violini* \\ Forschungszentrum Julich GmbH, Julich Centre for Neutron Science, Leo Brandt Strasse, D-52425 Julich, Germany and \\ Dipartimento di Fisica, Università di Perugia, Via Alessandro Pascoli, I-06123 Perugia, Italy \\ A. Orecchini, A. Paciaroni, C. Petrillo, and F. Sacchetti \\ Dipartimento di Fisica, Università di Perugia, Via Alessandro Pascoli, I-06123 Perugia, Italy, and Istituto Officina Materiali, \\ Unità di Perugia, clo Dipartimento di Fisica, Università di Perugia, Via Alessandro Pascoli, I-06123 Perugia, Italy
}

(Received 1 December 2011; revised manuscript received 14 March 2012; published 17 April 2012)

\begin{abstract}
The vibrational dynamics of vitreous glucose has been investigated by means of inelastic neutron scattering experiments, exploiting the coherent scattering cross section of deuterium in a fully deuterated sample and the high incoherent scattering cross section of hydrogen in a hydrogenated sample. The first part of the experiment allowed a rather detailed investigation of the collective dynamics in the $\mathrm{THz}$ range. The second part of the experiment was used to derive some information on the vibrational density of states of the system. The experiment confirms the presence of a propagating vibrational mode which is the natural extension at $\mathrm{THz}$ frequencies of the lower frequencies longitudinal sound mode. In addition, a second mode is also observed at a lower and almost constant frequency, showing an increasing intensity on reducing the wavelength. By comparing the dispersion relations of these collective modes to the experimental density of states, a possible relation between the low frequency mode and the well known excess of low frequency modes, that is, the boson peak, is identified.
\end{abstract}

DOI: 10.1103/PhysRevB.85.134204

PACS number(s): 61.05.fg, 61.43.-j, 63.50.Lm

\section{INTRODUCTION}

As is well known, glasses support long-wavelength sound waves as well as the corresponding crystalline materials do. This feature can be easily understood, because the microscopic arrangement is expected to become irrelevant if the medium can be assumed continuous. The continuum approximation holds in the case of longitudinal density fluctuations - sound waves - of hundreds of nm wavelengths, which can be probed by means of visible light scattering techniques in the wave-vector $Q$ range below $10^{-3} \mathrm{~nm}^{-1}$. On decreasing the wavelength, down to a few $\mathrm{nm}$ or even tenths of a nm, the density fluctuations directly probe the local interaction potential and the details of the disordered microscopic structure, so that the continuum approximation no longer holds. The density fluctuations at short wavelength have been investigated with inelastic x-ray (IXS) and neutron scattering (INS) techniques and numerically with molecular dynamics simulations (MD). The common findings obtained from the IXS and INS experiments on different glasses [e.g., v- $\mathrm{SiO}_{2}$ (Refs. 1,2), v- $\mathrm{GeO}_{2}$ (Ref. 3), 3-methylpentane, ${ }^{4}$ ethanol, ${ }^{5}$ and $\mathrm{GeSe}_{2}$ (Ref. 6)] enhance the presence of rather broad peaks in the total dynamic structure factor $S(Q, \omega)$ when it is measured at constant momentum transfer $Q$ as a function of the energy transfer $\hbar \omega$. The energy of these peaks follows a dispersion curve which is similar to that observed for the phonons in crystals when $Q$ is smaller than the position $Q_{p}$ of the first sharp diffraction peak (FSDP) of the static structure factor $S(Q)$. Of course this dispersion curve is isotropic, and the broadening is much larger than that normally observed in crystalline substances. The dispersion curve shows a linear slope at small wave vectors, which results in a propagation velocity usually slightly higher than the low frequency (say $1 \mathrm{MHz}$ ) sound velocity. In addition, a quasiperiodic trend of dispersion curves is often observed, ${ }^{5,6}$ which recalls the phonon dispersion beyond the first Brillouin zone in crystals. As to the behavior of the peak broadening on varying the momentum transfer, a $Q^{4}$ dependence below $10 \mathrm{~nm}^{-1}$ is observed $^{7}$ for both random-network and molecular glasses, while a $Q^{2}$ dependence of the line width is observed in the $\mathrm{nm}^{-1}$ range. ${ }^{8,9}$ These trends have been more recently enhanced in glycerol glass ${ }^{10}$ observing the transition between $Q^{4}$ and $Q^{2}$ trends at about $2 \mathrm{~nm}^{-1}$. The broadening of the peaks and its origin is one of the most debated characteristic of the glassy state and clearly indicates that these collective excitations are originated by a complex set of atomic or molecular motions linked to the intrinsic disorder of the system. Since the behavior of the collective excitations in glasses at the atomic length scale is far from being understood for what concerns both the characteristics of the dispersion relation and the trend of the damping, ${ }^{10}$ we believe that further investigation of other systems having different chemical and structural characteristics is necessary to identify in a wider range of systems specific and universal features of this complex state of condensed matter. In this context the class of carbohydrates seems to be quite promising and interesting. Indeed glasses of small carbohydrates are found in nature as protecting agents of biological assemblies and nucleic acids under circumstances of severe cold or drought. ${ }^{11,12}$ Their protecting effect consists of encapsulating the labile, reactive biopolymer in a rigid matrix of immobilized carbohydrate molecules. In the food and pharmaceutical industries, natural biopreservation is imitated. Disaccharides and oligosaccharides are used as encapsulating agents for flavors, medicines, and enzymes. In spite of their widespread use, very little is known about the molecular dynamics in glassy or near-glassy monosaccharides systems. The structure determination is available only in the case of glucose. ${ }^{13}$ Most available data are macroscopic, such as thermal expansivity, ${ }^{14}$ density,,${ }^{14}$ viscosity, ${ }^{15,16}$ fragility,,${ }^{17}$ and diffusion constants of small traces of water. ${ }^{18}$ The temperature dependent dielectric response has been also investigated so that some information on the size relaxation processes is now available. ${ }^{19}$ Nonetheless carbohydrates appear to form a 


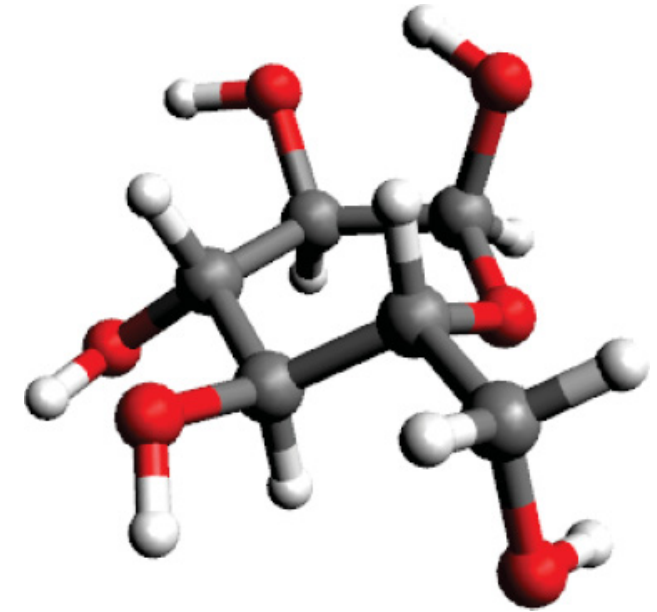

FIG. 1. (Color online) Glucose molecule picture.

class of very interesting glasses. Indeed, the axiomatic theory of ideally glassy networks has been recently broadened to include monosaccharides. ${ }^{20}$ If we consider also that the glass transition temperature is very close to room temperature, we understand that monosaccharides, and glucose, in particular, are of great interest for the study of glassy dynamics. Glucose has very well documented physical properties, is easily vitrified, and has a relatively simple molecular structure. Indeed, the glucose molecule, reported in Fig. 1, is rather small (chemical formula $\mathrm{C}_{6} \mathrm{H}_{12} \mathrm{O}_{6}$ ) and includes a one to one ratio between the carbon and oxygen atoms and the hydrogen atoms. In this condition the system is particularly suited for an investigation by means of inelastic neutron scattering. Thanks to the high coherent scattering length, an experiment performed on a fully deuterated sample of glucose allows the measurement of the dynamic structure factor $S(Q, \omega)$ and the study of the collective behavior in the small momentum transfer region. The collective dynamics can be also compared to the information obtained from incoherent neutron scattering of a hydrogenated sample which can be used to determine the vibrational density of states (DOS) projected on the hydrogen atoms. The paper is organized as follows: Sec. II is dedicated to the detailed description of the performed experiments, Sec. III describes the relevant aspects of data analysis and the outline of the main results, and Sec. IV is devoted to the conclusions.

\section{EXPERIMENT}

The sample used in the first part of the experiment was prepared starting from deuterated glucose purchased from Sigma Aldrich without further purification. To obtain the fully deuterated sample, the glucose powder was first diluted into $\mathrm{D}_{2} \mathrm{O}$ at room temperature and left for 2 days to properly substitute all residual exchangeable hydrogen atoms with deuterium. The solution was lyophilized under a vacuum in the presence of $\mathrm{P}_{2} \mathrm{O}_{5}$ for 2 days to achieve water content as low as possible. The powders were heated in a short time at $410 \mathrm{~K}$ and then rapidly cooled down to room temperature. After the cooling the sample was sealed to avoid any contact with the atmosphere and the consequent contamination by water. The transparent glass phase was produced directly in an aluminium container of hexagonal shape, to be used for the neutron scattering experiment. The sample was contained in seven cylinders of 12-mm diameter and 3-mm thickness, located at the corner and at the center of the hexagon. This container geometry allows an adequate decoupling of the various disks in order to reduce the multiple scattering contribution, and at the same time the individual sample quantity was large enough to produce a good quality glass phase. The second, fully hydrogenated sample was produced using a similar procedure but in a hollow cylindrical cell specifically designed to allow the insertion of a second inner hollow cylinder after the heating. In such a way, the sample was only contained in the 1-mm-thin gap between the two cylinders. The container was sealed directly at high temperature using a Viton O-ring which is perfectly stable at the sample preparation temperature. A good glass was produced also in this second case. The present experiment was twofold: a first one was devoted to the determination of $S(Q, \omega)$ and performed on a deuterated glucose sample using the INS time of flight (TOF) spectrometer BRISP installed at the High Flux Reactor of the Institut Laue Langevin (Grenoble, France). The measurements were performed at two temperatures: the first one at $298 \mathrm{~K}$, that is slightly below the glass transition $T_{g}=$ $311 \mathrm{~K}$ (Ref. 21), and the second one at $318 \mathrm{~K}$, that is slightly above the glass transition. The incoming wavelength was selected to be $0.9885 \AA$ corresponding to a neutron incoming energy of $83.6 \mathrm{meV}$. The elastic resolution, measured using the incoherent scattering from a vanadium standard, is Gaussian shaped with a full width at half maximum (FWHM) of $2.7 \mathrm{meV}$. The two-dimensional detector of $2 \mathrm{~m}^{2}$ of total active area, installed in a vacuum tank, was placed at a distance of $4 \mathrm{~m}$ from the sample. In this configuration, the minimum scattering angle was less than $1^{\circ}$ and the maximum scattering angle was about $13^{\circ}$. The sample was installed inside the vacuum line of the instrument, and the temperature was carefully and continuously controlled. The second experiment was devoted to the determination of the incoherent dynamic structure factor $S_{\text {inc }}(Q, \omega)$ which can provide useful information on the density of states of the system. The experiment was performed at $298 \mathrm{~K}$ using the IN4 spectrometer again located at the High Flux Reactor of the Institut Laue Langevin (Grenoble, France). The incident energy was fixed at $16.88 \mathrm{meV}$. The resulting elastic energy resolution is a Gaussian-shaped function with a FWHM of $1.2 \mathrm{meV}$. The minimum and maximum scattering angles were about $13^{\circ}$ and $120^{\circ}$, respectively. In both experiments the background was carefully determined by measuring the scattering from the empty sample containers and the same containers filled with a $\mathrm{Cd}$ full absorber. In this way we have been able to derive the sample scattering using the simple relationship:

$$
I(Q, \omega)=I_{s c}(Q, \omega)-T_{s}\left[I_{c}(Q, \omega)-I_{a}(Q, \omega)\right]-I_{a}(Q, \omega),
$$

where $I(Q, \omega)$ is the true intensity scattered from the sample, $I_{s c}(Q, \omega)$ is the intensity of the sample inside the container, $I_{c}(Q, \omega)$ is the intensity of the empty container, $I_{a}(Q, \omega)$ is the intensity of the container with the sample substituted by fully absorbing $\mathrm{Cd}$, and $T_{s}$ is the transmission of the sample which attenuates the sample container contribution. To derive the appropriate scattering from the sample, it is necessary to subtract the multiple scattering contribution. The multiple 


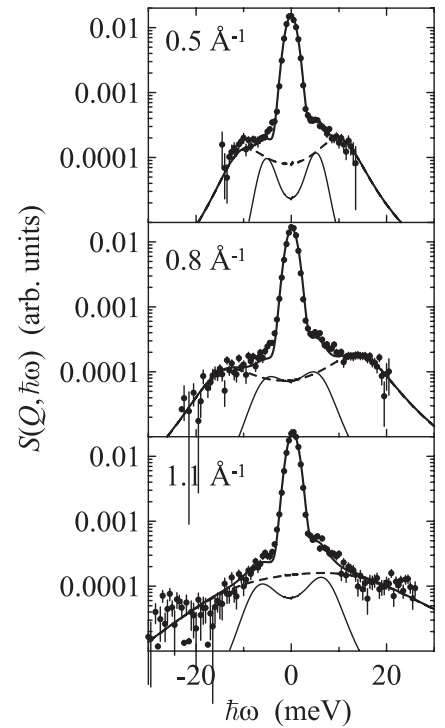

FIG. 2. Dynamic structure factor $S(Q, \hbar \omega)$, at selected values of the momentum transfer $Q$ (full dots). The thick full line is the result of the model fit discussed in the text. The dashed and thin lines are the high and low frequency inelastic contributions, respectively; see text. Notice the ordinate logarithmic scale used to enhance the low intensity inelastic contributions.

scattering contribution is obtained using an appropriate simulation procedure ${ }^{22}$ which is used also to determine the appropriate sample transmission $T_{s}$. The final results for the coherent intensity, measured at two temperatures just below and just above the glass transition temperature, were found to be temperature independent within the experimental errors. This result is expected since the fast dynamics in a region close to $T_{g}$ is related to atomic scale density fluctuations and no major structural change is present in this temperature region. The dynamic structure factor $S(Q, \omega)$ derived from the corrected coherent intensity, obtained by averaging the results measured at 298 and $318 \mathrm{~K}$, is presented in Fig. 2 at selected values of the momentum transfer $Q$. It is seen that the coherent intensity, which is proportional to the coherent structure factor $S_{c}(Q, \omega)$, shows an inelastic structure visible in all data with a well defined evolution on increasing the momentum transfer. The final results for the incoherent intensity are used to determine the incoherent structure factor $S_{\text {inc }}(Q, \omega)$ which is also reported in Fig. 3 where the data at a scattering angle of $16^{\circ}$ are presented. From $S_{\text {inc }}(Q, \omega)$ the vibrational density of states $g(\omega)$ can be obtained by extrapolating to low $Q$ the effective, $Q$ dependent, density of states $G_{\text {eff }}(Q, \omega)$ :

$$
G_{\text {eff }}(Q, \omega)=S_{\text {inc }}(Q, \omega) \frac{\omega}{[n(\omega)+1] Q^{2}},
$$

where $n(\omega)$ is the Bose factor. From $G_{\text {eff }}(Q, \omega)$ one can derive the density of states $g(\omega)$ by extrapolating to zero momentum transfer, using the incoherent scattering approximation. In the present sample, due to the high hydrogen content, the incoherent scattering approximation holds quite well, while the extrapolation to zero $Q$ is a quite difficult task. For the present purpose we content ourselves to the average of $G_{\text {eff }}(Q, \omega)$ in the low $Q$ region by limiting the scattering angle to a maximum value of $21^{\circ}$. A plot of $g(\omega)$ obtained in this way is reported in

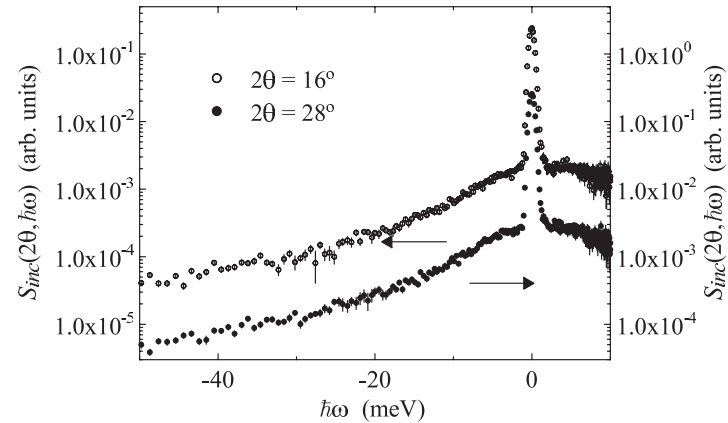

FIG. 3. Incoherent dynamic structure factor $S_{\text {inc }}(Q, \hbar \omega)$ at two selected scattering angles. The logarithmic ordinate scale is used to enhance the low intensity inelastic contributions.

Fig. 4. The translational peak, extending up to several tens of $\mathrm{meV}$, is well defined while the molecular peaks are visible on top of broad contributions. For comparison purposes the peaks observed in a Raman scattering experiment are also reported. ${ }^{24}$ In the inset, the reduced density of states $g_{\text {red }}(\omega)=g(\omega) / \omega^{2}$ is also shown, together with the expected Debye level (horizontal dashed line), that is quite low as compared to the actual $g_{\text {red }}(\omega)$. Looking at the general trends visible in the present experiments, we first observe that the spectra measured on BRISP show the presence of two inelastic structures at low wave-vector transfer and, on increasing $Q$, the higher energy inelastic component becomes broader and broader. The highfrequency structure can be reasonably associated to the evolution of the longitudinal acoustic mode in the $\mathrm{THz}$ frequency range which is explored in the neutron experiments. The lowfrequency peak is better seen at the intermediate wave-vector transfer. This peak is located at an almost constant energy, in an energy region typical of the boson peak (BP), as it will be also shown from the more detailed analysis. The comparison with experimental results on other systems and with moleculardynamics simulations ${ }^{7,8,23}$ suggests the interpretation of this low-frequency feature as the high-frequency evolution of the transverse-acoustic mode. ${ }^{25}$ However, it should be noted that

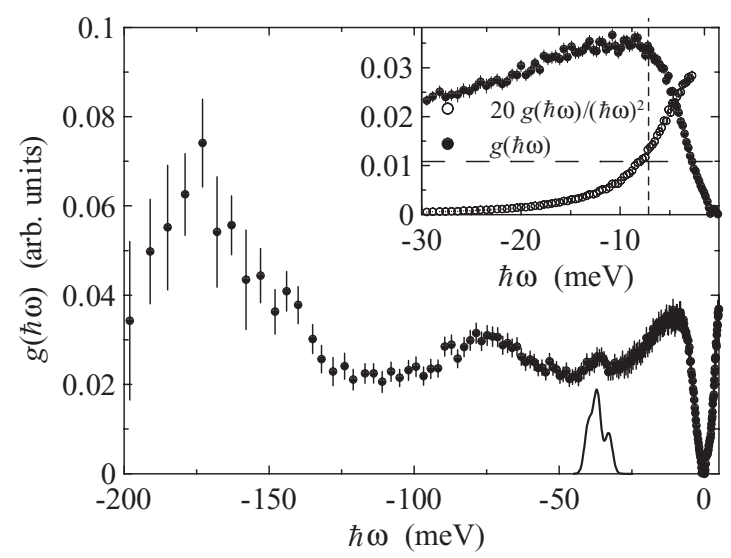

FIG. 4. Experimental density of vibrational states as derived from the experimental $S_{\text {inc }}(Q, \hbar \omega)$ (full dots); see text. The full line provides a guess for the information derived from the Raman spectroscopy data of Ref. 24. In the inset we report the reduced density of states $g_{\text {red }}(\hbar \omega)=g(\hbar \omega) /(\hbar \omega)^{2}$ in a smaller energy region. The horizontal dashed line is an estimate of the Debye contribution to $g_{\text {red }}(\hbar \omega)$. 
the density fluctuation modes loose their symmetry as the wave vector is increased, so that the longitudinal and transverse terms have not a well defined significance. Accordingly, the visibility of a transverse excitation in the intrinsically longitudinal density fluctuation spectra of a disordered system can be justified by the mixing phenomenon, ${ }^{26}$ or, in other words, at high frequency the transverse dynamics acquire a longitudinal component, observable in the neutron and x-ray scattering experiments. The incoherent scattering experiment does not provide a direct insight on the behavior of glassy glucose apart form the presence of the translational modes up to more than $20 \mathrm{meV}$, as it is also seen in the coherent scattering experiment.

\section{DATA ANALYSIS AND RESULTS}

The data have been analyzed using a standard empiric model in order to have a more quantitative analysis and to compare the results to the experimental density of states obtained from the incoherent scattering experiment. In order to determine the position and width of the system modes, all the spectra have been fitted to a model function of the following form which is based on the damped harmonic oscillator (DHO) which provides a good approximation in describing the collective modes of glasses:

$$
I(Q, \omega)=\left[I_{0}(Q) R(\omega)\right] \otimes\left[S_{\mathrm{emp}}(Q, \omega) \frac{\hbar \omega}{k_{B} T}[n(\omega)+1]\right],
$$

where the model function for the dynamic structure factor is convoluted with the instrumental resolution function $R(\omega)$ which is practically $Q$ independent. The final empiric model is composed of an elastic line and two DHO contributions which account for the inelastic contributions:

$$
\begin{aligned}
S_{\mathrm{emp}}(Q, \omega)= & A_{e l}(Q) \delta(\omega) \\
& +\frac{A_{L}(Q)}{\pi} \frac{\Omega_{L}^{2}(Q) \Gamma_{L}(Q)}{\left[\omega^{2}-\Omega_{L}^{2}(Q)\right]^{2}+\omega^{2} \Gamma_{L}^{2}(Q)} \\
& +\frac{A_{H}(Q)}{\pi} \frac{\Omega_{H}^{2}(Q) \Gamma_{H}(Q)}{\left[\omega^{2}-\Omega_{H}^{2}(Q)\right]^{2}+\omega^{2} \Gamma_{H}^{2}(Q)},
\end{aligned}
$$

where the subscripts refer to low $(L)$ and high $(H)$ frequency components. The presence in Eq. (2) of one elastic and two inelastic components determines the presence of seven fitting parameters, that is, $A_{e l}(Q), A_{L}(Q), A_{H}(Q), \Omega_{L}(Q), \Omega_{H}(Q)$, $\Gamma_{L}(Q)$, and $\Gamma_{H}(Q)$. Despite the number of parameters the fit was stable enough to provide a good determination of all of them because of the fortunate circumstance that the two modes have a different frequency and the low one is far enough from the quasielastic peak. The result of the fitting procedure is also reported in Fig. 2 in comparison to the experimental data and on a logarithmic scale to enhance the low intensity inelastic tails. The two fitted inelastic components were also reported in the figure to show the relative importance of the two components. The DHO frequencies $\Omega_{L}(Q)$ and $\Omega_{H}(Q)$ are reported in Fig. 5 together with the corresponding damping parameters $\Gamma_{L}(Q)$ and $\Gamma_{H}(Q)$. The experimental dispersion curves go through a break at $Q \simeq 0.6 \AA^{-1}$, where a small peak is visible in the static structure factor $S(Q)$ obtained by means of integration on $\omega$ of the experimental $S(Q, \omega)$ from

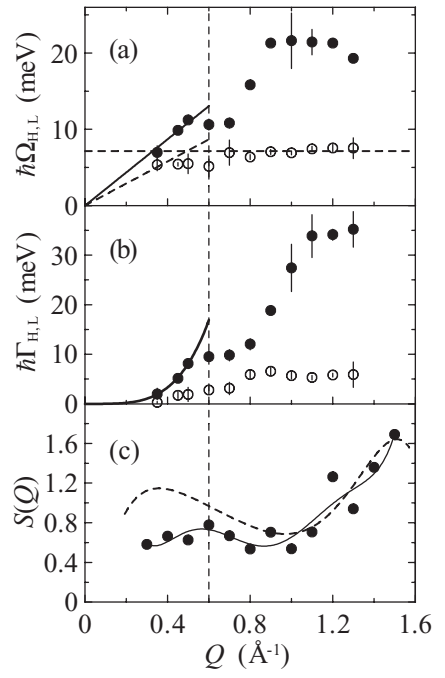

FIG. 5. (a) Dispersion relations of low $L$ (empty circles) and high $H$ (full dots) frequency modes, as derived from the fit described in the text. The full line is the linear fit to the low $Q$ portion of the dispersion relation, while the dashed line describes the dispersion derived from the low frequency sound velocity. The horizontal dashed line is the average of $\hbar \Omega_{L}(Q)$ when $Q$ is in excess of $0.5 \AA^{-1}$. (b) Damping parameters $\hbar \Gamma_{L}(Q)$ (empty circles) and $\hbar \Gamma_{H}(Q)$ (full dots). The full line is a quartic fit to the low $Q$ data. (c) Static structure factor $S(Q)$ as derived by integrating the present dynamic structure factor $S(Q, \omega)$ after the subtraction of the incoherent contribution. The thin line is a guide to eyes, while the dashed line is $S(Q)$ from the data of a partially deuterated sample. ${ }^{13}$ The vertical dashed line indicates the position of the first peak of the static structure factor $S(Q)$ as determined from the present data; see text.

the present data. This behavior is quite interesting because it indicates that the medium range order, identified by this peak of $S(Q)$, has an effect on the glasses dynamics in a similar way to what happens in crystals. One can also observe that the damping factor $\Gamma_{H}(Q)$ follows a trend similar to the corresponding frequency $\Omega_{H}(Q)$. On the contrary $\Omega_{L}(Q)$ has an almost opposite trend with respect $\Omega_{H}(Q)$, even if $\Omega_{L}(Q)$ is almost constant. Unfortunately $\Gamma_{L}(Q)$ is too small to derive a good information on its trend at low $Q$ also because the strength of the $L$ mode is negligible in this momentum range. On the contrary the accuracy in determining $\Gamma_{H}(Q)$ is good enough to establish that it behaves like $Q^{4}$ when $Q \leqslant 0.5 \AA^{-1}$. Since the local structure evolves already at $Q \simeq 0.7 \AA^{-1}$ the data do not allow us to discuss a possible transition $^{7,10}$ from a low $Q$ behavior like $Q^{4}$ to a higher $Q$ one like $Q^{2}$. Looking at the peak of $S(Q)$, observed in the partially deuterated sample, ${ }^{13}$ it is seen that the low $Q$ peak is more intense and shifted at a smaller momentum than in the present case. This behavior suggests that the peak is related to the deuterium-hydrogen in the partially substituted sample-atoms correlation. Although the small $Q$ peak is not fully reliable in the diffraction experiment, ${ }^{13}$ its intensity suggests that it must be related to the hydrogen atoms in the $\mathrm{CH}_{2}$ chain because its intensity is much different from the present one in the fully deuterated sample. Further insight into the behavior of the collective modes is obtained from the phase velocity of the $H$ mode $v_{H}(Q)=\Omega_{H}(Q) / Q$, which is reported in Fig. 6 . It is readily seen that, on increasing $Q, v_{H}(Q)$ increases 


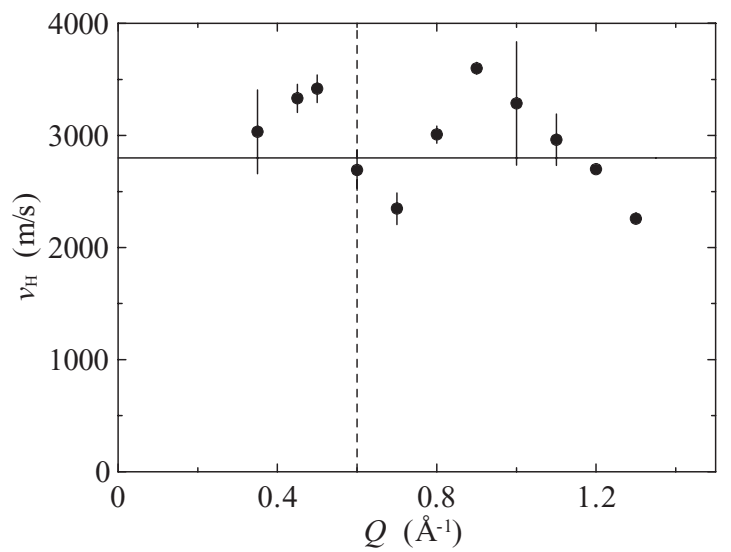

FIG. 6. Phase velocity $\Omega_{H}(Q) / Q$ from the present data (full dots) as compared to the low frequency sound velocity (thin line). The vertical dashed line indicates the position of the first peak of the static structure factor $\mathrm{S}(\mathrm{Q})$ as determined from the present data; see text.

as compared to the low frequency sound velocity and then decreases as the momentum approaches the position of the first peak of $S(Q)$. This behavior is strongly consistent with the nature of a longitudinal collective mode and provides a further evidence on the nature of the $H$ mode. The maximum velocity of the mode is obtained at $Q \simeq 0.5 \AA^{-1}$ indicating some evolution from the low frequency region to the high frequency one where the structure starts to affect the mode dispersion curve. In Fig. 7 we report the ratios $\Gamma_{L, H}(Q) / \Omega_{L, H}(Q)$ as a function of the momentum transfer. These ratios show first that the high energy mode becomes overdamped, that is, $\Gamma_{H}(Q) \geqslant \Omega_{H}(Q)$ when $Q \geqslant 0.9 \AA^{-1}$, that is a position close to the first diffraction peak of $S(Q)$, whereas the $L$ mode is never overdamped and shows a narrowing as $Q$ approaches the position of the principal peak of $S(Q)^{13}$ at $Q \simeq 1.5 \AA^{-1}$. This behavior is typical of a propagating mode in crystals as the momentum transfer approaches a reciprocal lattice point, thus supporting the idea that the first diffraction peaks are related

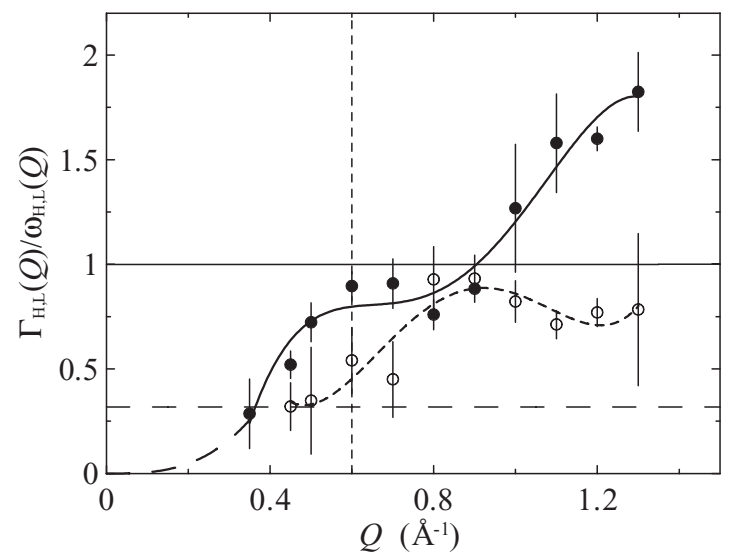

FIG. 7. Ratio between the damping parameters and the corresponding frequencies $\Gamma_{H}(Q) / \Omega_{H}(Q)$ (full dots) and $\Gamma_{L}(Q) / \Omega_{L}(Q)$ (empty circles) versus $Q$. The full and dashed lines are guides to eyes while the long dashed line is deduced from the low $Q$ fits of $\Gamma_{H}(Q)$ and $\Omega_{H}(Q)$. The vertical dashed line indicates the position of the first peak of the static structure factor $\mathrm{S}(\mathrm{Q})$ as determined from the present data; see text.

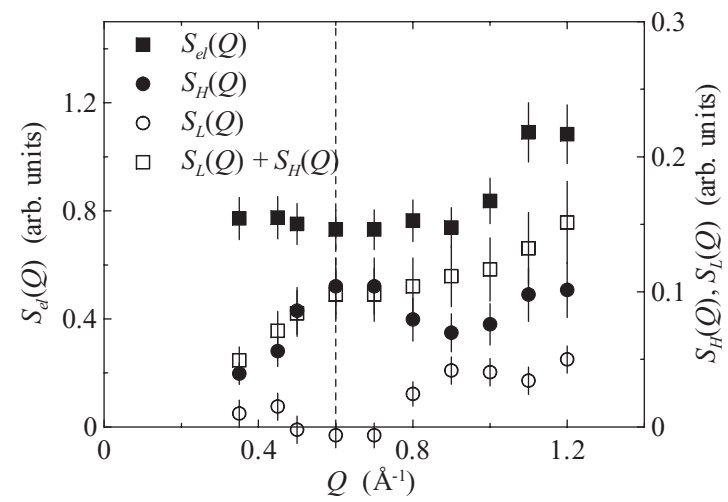

FIG. 8. Intensities of the elastic (full squares), high (full circles), and low (empty circles) frequency components of the fitting model function, used to fit the experimental dynamic structure factor. The sum of low and high intensities is also reported (empty squares). The vertical dashed line indicates the position of the first peak of the static structure factor $\mathrm{S}(\mathrm{Q})$ as determined from the present data; see text.

to a medium range structure which affects also the system dynamics. Additional information about the nature of the $L$ mode is derived looking at Fig. 8, where the intensities of the $L, H$, and elastic peaks are reported, as they are obtained by integration in energy of the relating components in the fitting model function of Eq. (4). It is very interesting to observe that the $L$ mode shows low intensity at low $Q$ and a minimum at the position of the peak of $S(Q)$. The different nature of the $L$ mode with respect to $H$ mode is evident, because the lower intensity at low $Q$ in the case of the $L$ mode indicates that this mode produces small longitudinal density fluctuations which increase on increasing $Q$. The $L$ mode can be either opticlike or acoustic transverselike, but more information can be gained only by exploring the dispersion relation on decreasing $Q$ below the present range. Unfortunately such an experiment is quite challenging because one needs at the same time a sharp energy resolution and a wide dynamic range, while the $L$ mode intensity is decreasing thus reducing its visibility. At present there is almost no chance of exploring this region of the $(Q, \omega)$ space. In order to give a better understanding of the dispersion curve behavior, the present results can be compared to predictions from previous simulation work. ${ }^{27}$ First of all we observe that the dispersion curves obtained from the simulated dynamic structure factors of the longitudinal and transverse fluctuations show a linear trend in the low $Q$ region up to the Ioffe-Regel limit and beyond. This behavior is also found in the present experiment for the $H$ mode, which is interpreted as the high frequency longitudinal collective mode. The $H$ dispersion curve found in our experiment shows a clear deviation from the linear trend at wave-vector transfer larger than the Ioffe-Regel wave vector and comparable to the position of a peak in the $S(Q)$. Thus the deviation from linearity is due to a length scale associated to the structure, that is larger than the length scale associated to the Ioffe-Regel crossover, but still smaller than that associated to the FSDP in the $\mathrm{S}(\mathrm{Q})$. Therefore the correlations responsible for this peak-as well as of the deviation from linearity - must be related to positions of pseudo-ordered atoms on a larger distance with respect to the average atomic distance responsible for the FSDP. The hydrogen chain, which can give rise to an extended bond 


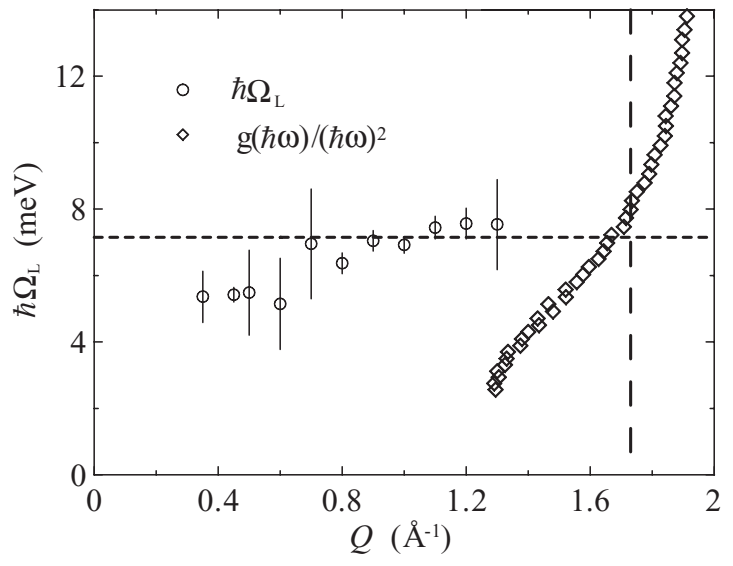

FIG. 9. Comparison between the dispersion relation $\hbar \Omega_{L}(Q)$ of the low frequency mode (empty circles) and the reduced density of vibrational states $g(\hbar \omega) /(\hbar \omega)^{2}$ (diamonds).

network, seems to be the likely responsible in the formation of this medium range order. Contrary to the findings of the simulation, ${ }^{27}$ the $L$ mode of the present experiment behaves like a low lying mode observed in strong glasses $\mathrm{GeO}_{2}$ (Ref. 3) and $\mathrm{GeSe}_{2}$ (Ref. 6), and it shows an almost constant frequency. Nonetheless the energy position of the Ioffe-Regel limit of the $L$ mode is about $5 \mathrm{meV}$, a value similar to the position of the boson peak as in the case of the simulation, ${ }^{27}$ whereas for the $H$ mode it is about $11 \mathrm{meV}$, much higher than the position of the boson peak. These results support a picture where the $L$ mode is related to the transverse density fluctuations, which have also a longitudinal component visible in the neutron scattering experiment, but for a more definitive conclusion a lower $Q$ region must be investigated, even if a very challenging experiment is necessary to possibly distinguish between an opticlike mode and an acoustic mode. Finally the incoherent scattering experiment provides some information about the density of states of the present system, which can be useful to better understand some crucial aspects. In particular we determined the reduced density of states $g_{\text {red }}(\omega)=g(\omega) / \omega^{2}$, that is a direct probe of the excess of vibrational states with respect to the Debye level, i.e., the boson peak. The present $g_{\text {red }}(\omega)$ is shown in the inset of Fig. 4 and in Fig. 9, where it is seen there is an increase in the low energy region, although a clear peak is not visible. It is not straightforward to stress two relevant aspects: at the frequency corresponding to the $L$ mode, it is seen that the reduced density of states probes a clear slope variation and crosses the expected Debye level. The interpretation of this features is very speculative at this stage. Indeed the reduced density of states defines the second inverse moment of the density of states, which weights heavily the lowest frequency excitations, including also the $L$ mode. Moreover, at high temperature, the second inverse moment gives the main contribution to the measurements of the mean square displacement from the equilibrium position of atoms or molecules involved in collective vibrations. Thus the visibility of a change in the slope of the reduced density of states might be interpreted as the sign of an increasing mean square displacement at the lowest frequencies, where we found the boson peak and the $L$ mode. Such a behavior is already been shown ${ }^{28}$ in chalcogenide glasses, where the authors also suggest that the second inverse moment might be sensitive to intermediate range structure in those glasses. For a straightforward comparison of $g_{\text {red }}(\omega)$ to the $L$ mode dispersion relation, we report them on the same plot in Fig. 9, where we see a rather convincing overlap between $g_{\text {red }}(\omega)$ and $\Omega_{L}(Q)$. It supports the idea that the boson peak is due to the presence of an additional low energy mode having an almost $Q$ independent dispersion relation.

\section{CONCLUSIONS}

In this work we presented a detailed study of the highfrequency dynamics in vitreous glucose. The INS experiments here presented provide some accurate information on the presence of two rather well defined modes, one having a low and almost $Q$ independent energy and one having the characteristics of a longitudinal acoustic mode. Thanks to the fortunate circumstance that the position of the low lying mode is fairly high, the position and the width of two modes in a broad $Q$ region, below and above the first sharp diffraction peak, are analyzed using a single instrument. These findings parallel the results already obtained in strong glasses. ${ }^{6}$ Here, in the investigated $Q$ range, the low-frequency mode shows a rather flat dispersion curve, and it is located in the energy range of the boson peak. As seen in all other glasses, the high-frequency mode shows typical features of an acoustic mode and a rather well defined dispersion curve is obtained in a wide $Q$ interval. The dispersion relation has a principal maximum at $Q \approx Q_{P} / 2$, then it goes toward a minimum at $Q_{P}$. The width shows a similar pseudoperiodicity as the dispersion curve. Nonetheless, the $H$ mode dispersion curve shows a secondary minimum which seems to be related to the first diffraction peak in a position where also the damping has a visible decrease. The pseudoperiodic behavior confirms the previous suggestion that the wave vectors corresponding to the first diffraction peaks act as pseudoreciprocal lattice ${ }^{6}$ vectors for the glass, thus defining pseudo-Brillouin zones. The evidences from this and previous works suggest the existence of a medium-range order in topologically disordered systems, which has some similarity to the local order present in the crystalline phase within the unit cell. The vibrational modes probed with INS are characterized by a width comparable to their position and with the same periodicity. Therefore peaks in the dynamic structure factor should be considered as the spectral densities of atomic motions intrinsically disorder allowed. The results obtained on glucose are in agreement with the tendency of other glasses and suggest the ability of the hydrogen chain in the formation of a limited order in the nanometric range in the glassy state as it is the case of other glasses having well defined directional bonds. The findings for glucose open the possibility of further research in this context, in order to link the existence of low-frequency modes at almost constant energy, the fragility and the medium-range order of these systems. The specific role of the hydrogen chain must be better understood, exploring the dynamics on increasing the temperature to observe the transition from the arrested state and the undercooled phase.

\section{ACKNOWLEDGMENTS}

We acknowledge the ILL for providing neutron beam time. 
*n.violini@fz-juelich.de

${ }^{1}$ B. Ruzicka, T. Scopigno, S. Caponi, A. Fontana, O. Pilla, P. Giura, G. Monaco, E. Pontecorvo, G. Ruocco, and F. Sette, Phys. Rev. B 69, 100201 (2004).

${ }^{2}$ G. Baldi, V. M. Giordano, G. Monaco, F. Sette, E. Fabiani, A. Fontana, and G. Ruocco, Phys. Rev. B 77, 214309 (2008).

${ }^{3}$ L. E. Bove, E. Fabiani, A. Fontana, F. Paoletti, C. Petrillo, O. Pilla, and I. C. V. Bento, Europhysics Lett. 71, 563 (2005).

${ }^{4}$ G. Baldi, A. Fontana, and A. Giugni (unpublished).

${ }^{5}$ A. Matic, C. Masciovecchio, D. Engberg, G. Monaco, L. Börjesson, S. C. Santucci, and R. Verbeni, Phys. Rev. Lett. 93, 145502 (2004).

${ }^{6}$ L. Orsingher, G. Baldi, A. Fontana, L. E. Bove, T. Unruh, A. Orecchini, C. Petrillo, N. Violini, and F. Sacchetti, Phys. Rev. B 82, 115201 (2010) and references therein.

${ }^{7}$ B. Rufflé, G. Guimbrètiere, E. Courtens, R. Vacher, and G. Monaco, Phys. Rev. Lett. 96, 045502 (2006).

${ }^{8}$ F. Sette, M. H. Krish, C. Masciovecchio, G. Ruocco, and G. Monaco, Science 280, 1550 (1998).

${ }^{9}$ A. Matic, D. Engberg, C. Masciovecchio, and L. Borjesson, Phys. Rev. Lett. 86, 3803 (2001); A. Matic, L. Borjesson, G. Ruocco, C. Masciovecchio, A. Mermet, F. Sette, and R. Verbeni, Europhys. Lett. 54, 77 (2001).

${ }^{10}$ G. Monaco and S. Mossa, Proc. Natl. Acad. Sci. USA 106, 3659 (2009).

${ }^{11}$ J. H. Crowe and J. S. Clegg, Anhydrobiosis (Dowden, Hutchinson, \& Ross, Stroudsburg, PA, 1973).

${ }^{12}$ C. A. Angell, Science 267, 1924 (1995).

${ }^{13}$ R. H. Tromp, R. Parker, and S. G. Ring, J. Chem. Phys. 107, 6038 (1997).
${ }^{14}$ G. S. Parks, H. M. Huffman, and F. R. Cattoir, J. Phys. Chem. 32, 1366 (1928).

${ }^{15}$ G. S. Parks, L. E. Barton, M. E. Spagt, and J. W. Richardson, J. Phys. 5, 193 (1934).

${ }^{16}$ A.-L. Ollett and R. Parker, J. Texture Stud. 21, 355 (1990).

${ }^{17}$ C. A. Angell, R. D. Bressel, J. L. Green, H. Kanno, M. Oguni, and E. J. Sare, J. Food Eng. 22, 115 (1994).

${ }^{18}$ R. Parker and S. G. Ring, Carbohydr. Res. 273, 147 (1995); R. H. Tromp, R. Parker, and S. G. Ring, ibid. 303, 199 (1997).

${ }^{19}$ T. R. Noel, R. Parker, and S. G. Ring, Carbohydr. Res. 282, 193 (1996).

${ }^{20}$ J. C. Phillips, Phys. Rev. B 73, 024210 (2006).

${ }^{21}$ R. Wungtanagorn and S. J. Schmidt, J. Therm. Anal.: Calorimetry 65, 9 (2001).

${ }^{22}$ C. Petrillo and F. Sacchetti, Acta Cryst. A 46, 440 (1990); Acta Crystallogr. Sect. A 48, 508 (1992).

${ }^{23}$ S. N. Taraskin, Y. L. Loh, G. Natarajan, and S. R. Elliott, Phys. Rev. Lett. 86, 1255 (2001).

${ }^{24}$ S. Söderhölm, Y. H. Roos, N. Meinander, and M. Hotokka, J. Raman Spectrosc. 30, 1009 (1999).

${ }^{25}$ U. Buchenau, M. Prager, N. Nucker, A. J. Dianoux, N. Ahmad, and W. A. Phillips, Phys. Rev. B 34, 5665 (1986).

${ }^{26}$ M. Sampoli, G. Ruocco, and F. Sette, Phys. Rev. Lett. 79, 1678 (1997).

${ }^{27}$ H. Shintani and H. Tanaka, Nat. Mater. 7, 870 (2008).

${ }^{28}$ P. Boolchand, R. N. Enzweiler, R. L. Cappelletti, W. A. Kamitakahara, Y. Cai, and M. F. Thorpe, Solid State Ionics 39, 81 (1990). 